International Journal of Engineering \& Technology, 7 (2.7) (2018) 868-871
International Journal of Engineering \& Technology
WPC
Website: www.sciencepubco.com/index.php/IJET
Research Paper

\title{
Implementation Of Hybrid Scheduler In Hadoop
}

\author{
B Thirumala Rao*, M Susmitha ${ }^{2}$, T Swathi ${ }^{2}$, G Akhil ${ }^{2}$ \\ 1 Professor, Koneru Lakshmiah Educational Foundation,Department of CSE,India \\ 2 Student, Koneru Lakshmiah Educational Foundation,Department of CSE, India \\ *Corresponding author E-mail: susmisweety.1@gmail.com
}

\begin{abstract}
The paper focusses on priority based round robin scheduling algorithm for scheduling jobs in Hadoop environment. By Using this Proposed Scheduling Algorithm it reduces the starvation of jobs. And the advantage of priority scheduling is that the process with the highest priority will be executed first. Combining the both strategies of round robin and priority scheduling algorithm a optimized algorithm is to be implemented. Which works more efficiently even after considering all the parameters of scheduling algorithm. This proposed algorithm is also compared with existing round robin and priority scheduling algorithms.
\end{abstract}

Keywords: Efficiency, Gantt chart, parameters, Priority scheduling algorithm, Round Robin Scheduling algorithm.

\section{Introduction}

Hadoop is an open-source system that permit to store and process data in a distributed environment across combination of computers utilizing basic programming models. Hadoop is also used to perform operations like local Computations and Storage. It has two noteworthy Components:

1. Computation layer(Map Reduce).

2. Storage layer (Hadoop Distributed File System).

A Hadoop Architecture Contains Namenode, Datanode Secondary Name node.The Hadoop Distributed File System (HDFS) is based on the Google File System (GFS) and provides a distributed environment that is designed to run on commodity based hardware. It is highly fault-tolerant and also used for a hardware that is available cheaply. It provides high throughput access to application related data and is suitable for applications that are having large data sets. Apart from the abovementioned two noteworthy Components, It also has other two modules:

Hadoop Common: These are Java libraries and are used even by other Hadoop modules.

Hadoop YARN: It is a Open-Source structure for job scheduling and also cluster resource management.

They are different elements of Hadoop they are Namenode, Datanode, Job Tracker, Task Tracker, Mapreduce.

Namenode: Namenode is the master in the master/slave architecture. It stores all the files on the Hadoop disturbed file system (HDFS) and keeps track of all the files. All the data will be present in namenode itself. It doesn't store he data of itself. In case if the namenode is down, then the secondary namenode takes charge of the primary name node. Both the primary name node and secondary namenode are given an equal amount of space. Datanode: Datanode is the slave of the master node which is the namenode. The default replication factor and block size of datanode is 3 and $64 \mathrm{MB}$ respectively. It Connects to the NameNode and the Data is send from the NameNode to DataNode. When the Namenode needs data it requests the data from the Datanode.

JobTracker: This process executes on each node separately and not usually on a data node. Job tracker interacts with the name node to know the location of the data. Job tracker monitors the individual task trackers and it will submits back the overall status of the job back to the client. When the job tracker is down, HDFS will still be working, but the MapReduce execution cannot start and the existing MapReduce jobs will be stopped.

Task tracker: Task tracker runs on all the data nodes. Mapper and Reducer tasks are also executed on the data node. Task tracker will be in constant interaction with the job tracker signaling the progress of the task in execution. Task tracker failure is not considered fatal. When the task tracker doesn't respond, then the Job tracker will assign the task executed by the task tracker to any other node.

Map Reduce: The job of a Map Reduce is to divide data into seperate data sets. Then they are processed by the map tasks in a simultaneous manner. The structure sorts the outputs generated by maps, They are now inputs to the reduce tasks. Both the input and output of the jobs are kept in the form of a file. The structure takes care of scheduling tasks, monitors them and re-executes the failed tasks. In map reduce values are stored in the form of <key, value> pair.

Secondary Name Node is used to Retrieve data from all the DataNodes if the Primary NameNode halts down.

\section{Scheduling}

Scheduling is the method by which work specified is assigned to the resources to complete the job. The work may include virtual computation of elements such as threads, processes which are inturn scheduled into the resources that are involved in hardware such as processors and network links. It is used to keep all the 
computer related resources busy, it will allow multiple users to share system resources effectively. The main objective of these algorithms is to reduce the Resource Starvation. And to ensure equality among the parties those are utilizing the resources. They are different types of schedulers like FIFO Scheduler, Roundrobin Scheduler, Priority Scheduler, Shortest Job First Scheduler etc.

By doing survey on all the schedulers we had come to a conclusion that every scheduler alone has its own advantages and disadvantages.

The default scheduling algorithm in Hadoop is FIFO Scheduler, in which the jobs that are present in the queue first are executed first. In FIFO scheduling algorithm all the process is given equal priority at the time of execution. But the major Disadvantage of FIFO scheduling is that the if the jobs with the lowest burst time arrive at the end of the queue then that particular process will be starved for long time.

Every scheduler has its own parameter to measure its efficiency like Round robin has Context Switches. The value of Context Switches should be as low as possible.

Larger Waiting Time for each process to complete its execution. But in Proposed Algorithm the waiting time of processes is very less compared to the process of execution of Round robin scheduling algorithm alone.

Throughput is the number of cycles executed per unit time. In Round robin the number of cycles executed are more. So this is the major disadvantage of round robin algorithm.

\section{Different types of scheduling}

FCFS (First come first serve) is a non preemptive scheduling algorithm. It works on the basis of queue. It assigns the priority in an order that is the process which requests the processor first for the execution. The process or the job which requests the CPU first is allocated the CPU first and if the other process has to wait until the CPU is free then all the arriving jobs after this job will be inserted into the rear or tail of the queue. The process which completed its execution will be removed from the head or front of the queue.

SJF is also a non pre-emptive scheduling algorithm. Among all the processes which one is having the shortest run time will get the high priority and run the job first. This Scheduling is mostly beneficial if waiting time is minimum for all the shorter jobs. This is more useful in case of batch jobs where runtime is known in advance.

Roundrobin is a preemptive scheduling algorithm. In Round robin scheduling all the processors are given an equal chance to execute. The main parameter of roundrobin scheduler is the time slice or quantum. Each process is executed only till the time slice and further next processes are executed. If the burst time is less than the time slice for a particular process, then that process completes execution and it is discarded from the queue. No of executions in a round robin is calculated based on the context switches.

In Priority scheduling all the process will be executed based on the priority number of that particular task. If the Priority number is higher for a process which has, the more burst time, then in that case priority scheduling is not recommended. Because the later process doesn't get a chance to execute the waiting time of remaining process will be more. Priority scheduling is recommended in a case where the process has, the less burst time value and the priority number is high.

Multi Level Queue :It implements scheduling algorithm.It is suitable is any type of job.In this type Jobs cannot navigate from one Queue to another. - Once a queue is allocated it should be in that queue itself. In foreground it is an interactive which follows round robin algorithm. In background it is a batch processing which follows FCFS scheduling algorithm. The main advantage of this scheduling algorithm is As Short CPU bounds are enabled and Prioritized and therefore it is processed quickly. The disadvantage is Monitoring of Queues is Compulsary which tends to be Costly. Multilevel feedback queue allows the obs o avigate from ne ueue to another. The advantage of this Scheduling algorithm is A process that waits for a long time in a lower priority queue then it may be moved to a higher priority queue. And Disadvantage is As the process should move around the Queue the CPU overhead will be more.

Fair Scheduling was developed by Facebook. and is used to assign resources to jobs such that on average over time, each job gets an equal share of the available resources. The result is that jobs that require less time are able to access the CPU and finish intermixed with the execution of jobs that require more time to execute. The advantage of fair scheduler is that it is simple to implement and is very efficient and Disadvantage of this scheduling algorithm is it has no concept of prioritize.

In Capacity scheduling, It was developed by Yahoo. Every Queue has Resources and follows FIFO strategy. The advantage of this scheduler is Optimal usage of resources. And the Disadvantage is that Users need know the system pretty well to set up configurations and choose proper queues well.

Delay Scheduling is the simple algorithm proposed by Zaharia et al. to address the conflict between locality and fairness.The advantage of this scheduling is Simplicity of scheduling.

SRTF (shortest remaining time first) is an Preemptive SJF The advantages of SRTF are short processes returns quickly and it has high yield jobs take some time to execute. The Disadvantage is Longer jobs may not get chance to execute if Shorter jobs are continuously added (Process Starvation).

Packet scheduling is the type of decision process which is used to decide the status of the packets whether they should be further proceeded or Dropped. Dropping of packets will be based on network. A scheduler will face problems in managing all the packets coming in because Each packet may have different Characteristics.

Process Scheduling is the combination of all the scheduling algorithms. They are no particular advantages or disadvantages for this Scheduling algorithm

\section{Scheduling Objectives}

For a scheduler to be working more efficiently then then the Throughput, CPU Utilization, Fairness of that particular scheduling algorithm should be high and the Waiting time, Response time, Context Switches must be as low as possible.

Table 4.1 Examining Schedulers using various parameters

\begin{tabular}{|c|c|c|c|c|c|}
\hline Sched uler & \begin{tabular}{|l|} 
CPU \\
overh ead
\end{tabular} & $\begin{array}{l}\text { Throu } \\
\text { ghput }\end{array}$ & $\begin{array}{l}\text { Turn } \\
\text { Arou nd } \\
\text { time }\end{array}$ & $\begin{array}{l}\text { Respo nse } \\
\text { time }\end{array}$ & Waiting time \\
\hline FCFS & $\checkmark$ & $\checkmark$ & $X$ & $\checkmark$ & $X$ \\
\hline SJF & - & $X$ & $\bullet$ & $\checkmark$ & $\checkmark$ \\
\hline Priori ty & - & $\checkmark$ & X & $X$ & . \\
\hline \begin{tabular}{|l|} 
Roun \\
d robin
\end{tabular} & $\mathrm{X}$ & - & $X$ & $\mathrm{X}$ & $\checkmark$ \\
\hline \begin{tabular}{|l} 
Multi \\
level \\
queue
\end{tabular} & $\mathrm{X}$ & $X$ & . & & • \\
\hline \begin{tabular}{|l} 
Capac \\
ity \\
\end{tabular} & $X$ & $X$ & $X$ & $X$ & $\checkmark$ \\
\hline Fair & 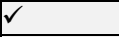 & $\mathbf{X}$ & $\mathrm{X}$ & $\mathrm{X}$ & $\checkmark$ \\
\hline Delay & - & $\checkmark$ & $\mathrm{X}$ & $X$ & $X$ \\
\hline \begin{tabular}{|l|} 
Work \\
Conse \\
rving
\end{tabular} & $\mathrm{X}$ & - & $\checkmark$ & $\checkmark$ & $\checkmark$ \\
\hline $\begin{array}{ll}\text { Netw } & \text { ork } X \\
\text { packe } & \\
\text { t }\end{array}$ & & $\checkmark$ & $\checkmark$ & $\mathrm{X}$ & $\checkmark$ \\
\hline \begin{tabular}{|l|} 
Earlie st \\
deadli ne \\
first
\end{tabular} & & $X$ & $\mathrm{X}$ & • & $\checkmark$ \\
\hline
\end{tabular}


The analysis of Scheduling algorithms based on the parameters: CPU Overhead:- CPU overhead measures the quantity of work a computer's central processing unit can perform and the percentage of that capacity that's used by individual computing tasks. Some of these tasks involve supporting the functions of the computer's operating system. (Or) Percent of time that the CPU is busy executing the process.

Throughput:- Throughput is that the number of jobs that are executed per unit time.

Turnaround time:- Turnaround time can be defined as the interval of time between submission of a process and its completion.

Response Time:- Response time can be defined as submission of requests and first response to the request.

Waiting Time:- Waiting time can be defined as the quantity of time the process spends in the ready queue waiting for the Central Processing Unit.

The overall analysis of a Scheduling algorithm is done by using the above parameters.

\section{Proposed algorithm}

We had proposed an algorithm by combining both strategies of Roundrobin and also the priority scheduling algorithm. In the proposed algorithm number of context switches will be reduced and then this algorithm will be the optimized algorithm.

We had considered an example and done analysis on each scheduler individually and recognized that combination of round robin and priority works even better.

Inorder to understand the proposed algorithm we should have a basic knowledge about Roundrobin and priority schedulers.

job generally specifies mapper,reducer.It communicates with resource manager.It supplies equipment to submit jobs.

The job is divided into map and reduce tasks for every node in the cluster to work .Both exist on the same set of nodes.It enables the job tracker program on nodes.

The job is to divide the input data into individual chunks.. The reduce task takes the data and combines the key value pairs. These tasks are executed only after the completion of the Map tasks.

A set of programs that run parallely, known as TaskTracker, monitor the status of each task. If a task fails,the Status of the job is reported to the Job Tracker. which reschedules the task on another node in the cluster.

This distribution of work enables map tasks and reduce tasks to run on smaller subsets of larger data sets, which ultimately provides maximum scalability.

The MapReduce Structure also increases parallelism by manipulating data that is stored across multiple clusters. It involves and verifies the data of the job.

Table 5.1 : Input table for a Scheduling Algorithm

\begin{tabular}{|l|l|l|}
\hline Jobs & Job Completion Time & Priority \\
\hline P1 & 20 & 4 \\
\hline P2 & 10 & 2 \\
\hline P3 & 6 & 5 \\
\hline P4 & 9 & 1 \\
\hline P5 & 15 & 3 \\
\hline
\end{tabular}

Example for Execution of Roundrobin Scheduling:

Consider the jobs and job completion time inorder to perform roundrobin scheduling algorithm on that processes. Time Slice is the important factor executing the jobs in the Roundrobin fashion. The output is represented in the form of a gnatt chart. Context Switches is the one which tells the no of executing steps that a process has taken to execute the job.

Suppose consider Time slice $=5$ then the completion time of process executes until completion of the job completion time and then same job is repeated. All the jobs are present in the form of a Queue. If all the Process complete execution of the job completion time once then again from starting the same process is repeated. Until the Completion of the Burst time of all the Processes.
In our algorithm we had overcame all the disadvantages of the Round robin scheduling algorithm they are :

1. Low Throughput.

2. Larger Waiting time.

3. Context Switches.

Based on some Parameters we can calculate the efficiency of the Scheduling.

Considering the Above Example:

No of Context Switches: 13

Average Waiting time: $33.2 \mathrm{~ms}$

TurnAroundtime: 45.2

Response Time: 10

Example for Execution of Priority Scheduling :

Consider the jobs that has the input values are Burst time and Priority.

All the jobs will be executed based on the Priority values of each jobs. The jobs with Highest Priority will be executed first.

The output of Priority Scheduling is Represented in the form of Gnatt Chart.

Considering Above Example:

Average Waiting time: $23.2 \mathrm{~ms}$

TurnAroundtime: 35.2

Response Time: 23.2

No of Context Switches is equal to number of jobs.

In priority scheduler the jobs with lowest priority may not get chance to execute and also they may be a chance of starvation of jobs. This is the major disadvantage of the Priority scheduling algorithm.

Example for Execution based on Proposed Algorithm:

In the Proposed Algorithm all the jobs should have a two input values they are Priority and Burst time.

They are two rounds inorder to execute the Proposed Algorithm they are:

We should even consider the TimeSlice value. For Example Consider the Timeslice $=5$.

Round 1: All the jobs are initially executed based on the priority scheduling.

In Round1, All the jobs are executed based on the Priority Scheduling and the process is executed until the timeslice value. So we had taken an example Considering Timeslice $=5$ so all the jobs completes a Burst time of 5 in Round1.

Round 2: Now the remaining burst time is considered in round 2 all the jobs in this round are executed based on the roundrobin scheduling technique.

By using this proposed algorithm we had overcomed all the disadvantages of Roundrobin and Priority Schedulers.

Considering Above Example:

No of Context Switches: 8

Average Waiting time: $13 \mathrm{~ms}$

TurnAroundtime: 20

Response Time: 8.2

Table 5.2 :Analysis of Schedulers against various parameters.

\begin{tabular}{|l|l|l|l|l|}
\hline Scheduler s & $\begin{array}{l}\text { No of } \\
\text { Context } \\
\text { Switche s }\end{array}$ & $\begin{array}{l}\text { Averag e } \\
\text { Waitin g } \\
\text { Time } \\
(\mathrm{ms})\end{array}$ & $\begin{array}{l}\text { Turn } \\
\text { Aroun d } \\
\text { Time }\end{array}$ & $\begin{array}{l}\text { Respons e } \\
\text { Time }\end{array}$ \\
\hline $\begin{array}{l}\text { Round } \\
\text { Robin }\end{array}$ & 13 & 33.2 & 45.2 & 10 \\
\hline Priority & 5 & 23.2 & 35.2 & 23.2 \\
\hline $\begin{array}{l}\text { proposed } \\
\text { Scheduler }\end{array}$ & 8 & 13 & 20 & 8.2 \\
\hline
\end{tabular}

The above table proves that the proposed algorithm works more efficiently compared to the existing techniques. Those ar the Round robin scheduling technique and Priority scheduling technique. 


\section{Conclusion}

By above results we had observed that the waiting time of Proposed Algorithm is less compared to the Roundrobin Algorithm and priority Schduling algorithm alone.

Even the Proposed algorithm is very optimized by considering different parameters like Context Switches, Turn Aroundtime, Response time.

The Proposed Algorithm is compared with the existing Round robin and Priority Schedulers it also overcomes the disadvantages that are present in both the scheduling algorithms. i.e; More number of Context Switches and Lowest priority job may not get chance to execute. Our proposed algorithm can be implemented in Real time systems inorder to increase the efficiency of the Existing Schedulers.

\section{References}

[1] kargahi, "A Method for Performance Analysis of EarliestDeadline-First Scheduling Policy" Proceedings of the 2004 International Conference on Dependable Systems and Networks (DSN'04) 0- 7695-2052-9/04 \$ 20.00 @ 2004 IEEE.

[2] Chakareski"In-Network Packet Scheduling and Rate Allocation: A Content Delivery Perspective" Jacob Chakareski IEEE TRANSACTIONS ON MULTIMEDIA, VOL. 13, NO. 5, OCTOBER 2011.

[3] Bagherinia"A novel algorithm for process scheduling in multiprocessor systems" 978-1-4673- 2225-6/12/\$31.00 @2012 IEEE.

[4] A. Fernandez "On the Isolation of Several Work-Conserving Scheduling Policies" 0- 7803-5794-9/99/\$10.00 01999 IEEE.

[5] H.S. Behera, Reena Kumari Naik "Improved Multilevel Feedback Queue Scheduling Using Dynamic Time Quantum and Its Performance Analysis " H.S. Behera et al, / (IJCSIT) International Journal of Computer Science and Information Technologies, Vol. 3 (2) , 2012,3801-3807.

[6] Yousi Zhen, Prasun Sinha, Ness B. hroff "Performance Analysis of Work-Conserving Schedulers for Minimizing Total FlowTime with Phase Precedence"

[7] Matei Zaharia "Delay Scheduling: A Simple Technique for Achieving Locality and Fairness in Cluster Scheduling".

[8] Nathanaël Cheriere" On the Usability of Shortest Remaining Time First Policy in Shared Hadoop Clusters".

[9] Iqra Sattar"Multi-Level Queue with Priority and Time Sharing for Real Time Scheduling".

[10] Yintian Wang et.al "A Round Robin with Multiple Feedback Job Scheduler in Hadoop" 978-1-4799-2030-3 /14/\$31.00 @2014 IEEE. 